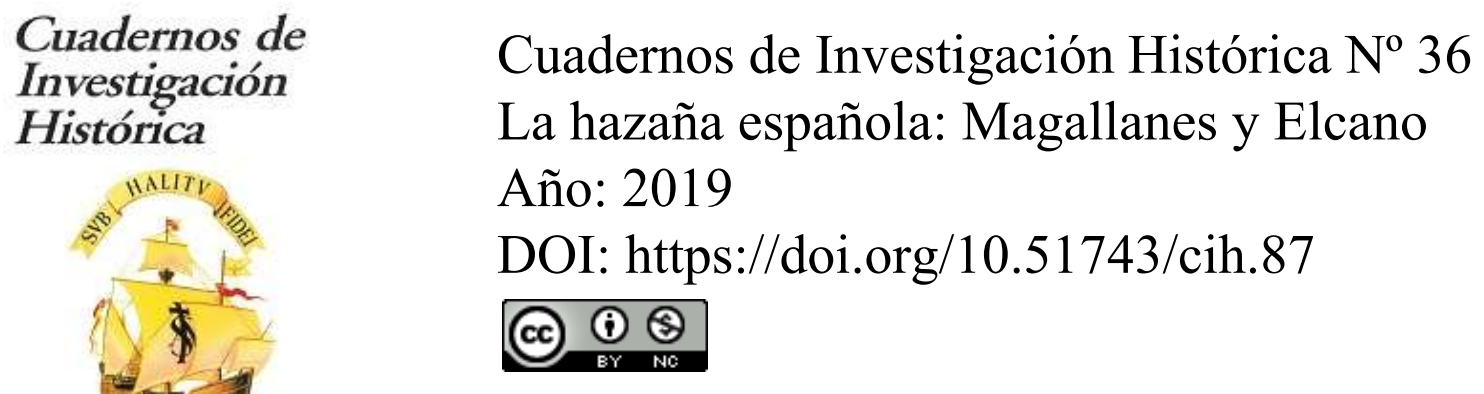

\title{
CIENCIA, NÁUTICA Y HOMBRES EN LA PRIMERA CIRCUNNAVEGACIÓN
}

SCIENCE, NAUTICS AND MEN IN THE FIRST CIRCUMNAVIGATION

\author{
ALEJANDRo KLECKER DE ELIZALDE
}

Escuela Internacional de Doctorado Universidad Rey Juan Carlos

\begin{abstract}
RESUMEN
Esta es la relación histórica de una ambición personal, la de Magallanes, y el apoyo decidido de varios personajes, de una visión comercial, la de la Corona y particulares e integrada por 265 historias personales. En ellas encontramos lo mejor y lo peor del ser humano: valentía, fuerza de voluntad, perseverancia, sentido espiritual, amor por lo desconocido, pasión por nuevas culturas, tierras, descubrimientos y también... el miedo, la traición, la cobardía, la codicia y la lujuria. Cada una de esas virtudes y defectos tienen nombres y apellidos propios. Los veremos en los episodios que jalonan esa aventura en su organización, financiación y resultados de la misma, cuyos principales hitos fueron: la gestación de la expedición en Sevilla Burgos, Valladolid... la preparación en Sevilla, la partida de Sanlúcar de Barrameda, la navegación hasta las Islas Canarias, Cabo Verde, Brasil, San Julián, el paso y el descubrimiento del ansiado Estrecho, que permitiera llegar a las islas de las especierías, objetivo último del viaje, la navegación por el Pacifico, el encuentro de las islas San Lázaro, la muerte de Magallanes y finalmente la vuelta.
\end{abstract}

Palabras clave: primera circunnavegación, Magallanes, Elcano 


\begin{abstract}
This is the historical relationship of a personal ambition, that of Magellan, and the determined support of various characters, of a commercial vision, that of the Crown and individuals and made up of 265 personal stories. In them we find the best and the worst of the human being: courage, willpower, perseverance, spiritual sense, love for the unknown, passion for new cultures, lands, discoveries and also... fear, betrayal, cowardice, greed and lust. Each of these virtues and defects have their own names and surnames. We will see them in the episodes that mark this adventure in its organization, financing and results, whose main milestones were: the gestation of the expedition in Seville Burgos, Valladolid ... the preparation in Seville, the departure from Sanlúcar de Barrameda, navigation to the Canary Islands, Cape Verde, Brazil, San Julián, the passage and the discovery of the long-awaited Strait, which would allow to reach the islands of spices, the ultimate objective of the trip, navigation through the Pacific, the meeting of the San Lázaro islands. , the death of Magellan and finally the return.
\end{abstract}

Keywords: first circumnavigation, magallanes, elcano

\title{
OBJETIVO DEL TRABAJO
}

— sta es la relación histórica de una ambición personal, la de Magallanes, y el Lapoyo decidido de varios personajes, de una visión comercial, la de la Corona y particulares e integrada por 265 historias personales. En ellas encontramos lo mejor y lo peor del ser humano: valentía, fuerza de voluntad, perseverancia, sentido espiritual, amor por lo desconocido, pasión por nuevas culturas, tierras, descubrimientos y también... el miedo, la traición, la cobardía, la codicia y la lujuria. Cada una de esas virtudes y defectos tienen nombres y apellidos propios. Los veremos en los episodios que jalonan esa aventura en su organización, financiación y resultados de la misma, cuyos principales hitos fueron: la gestación de la expedición en Sevilla Burgos, Valladolid... la preparación en Sevilla, la partida de Sanlúcar de Barrameda, la navegación hasta las Islas Canarias, Cabo Verde, Brasil, San Julián, el paso y el descubrimiento del ansiado Estrecho, que permitiera llegar a las islas de las especierías, objetivo último del viaje, la navegación por el Pacifico, el encuentro de las islas San Lázaro, la muerte de Magallanes y finalmente la vuelta.

Haremos una narración del estado de la ciencia náutica con sus instrumentos, elementos de navegación, y la cartografía, o mejor el arte de hacer mapas ( la ciencia cartográfica no sería reconocida como tal hasta el siglo XIX) previo y posterior a la hazaña.

Uno no puede sino estremecerse por esta colosal aventura, donde el hambre, el frío, la muerte, las tempestades, las depresivas calmas el miedo diario de la navegación y la persecución de los portugueses fueron una constante. Queda- 
mos admirados por la actitud de esos hombres, ante tantos retos y por su actitud de avidez cultural e interés por las nuevas culturas, razas o alimentos que fueron encontrando en su derrotero.

Fue una expedición comercial, diplomática, no de conquista o sometimiento. Respetuosa con los reinos a encontrar y con quién establecer lazos comerciales. Y muy cuidadosa con no infringir los Tratados con Portugal, como mencionaba el emperador Carlos $\mathrm{V}$ en sus cédulas y largas instrucciones, prodigio de organización y previsión de lo que pudieran encontrarse.

\section{LAS FUENTES}

Afortunadamente tenemos un certero conocimiento de lo ocurrido en la expedición, aunque todavía nos surgen algunas dudas que seguramente nunca serán aclaradas, salvo que aparecieran otros documentos en los archivos portugueses y españoles, que ya han sido estudiados en profundidad. Las fuentes accesibles son:

- Antonio Pigafetta, su diario. En el que ya sabemos que nunca citó a Elcano, seguramente por su papel en las iniciales amotinamientos o por mera antipatía. Es una narración antropológica, de costumbres, del carácter humano de los tripulantes, salpicado de anécdotas picantes o de comentarios a veces exagerados. Pero sin duda un entretenido diario de viaje. De Pigafetta cabe destacar que sufrió como el que más, fue herido, enfermó, pero consiguió completar el viaje. Un individuo portentosos sin duda.

El diario tuvo inicialmente cuatro ediciones hasta la definitiva de 1555, descubierta en 1800 en la Biblioteca Ambrosiana ${ }^{1}$.

- Diario de Francisco $\mathrm{Albo}^{2}$, Contramaestre de la Trinidad. Especialmente de interés por sus observaciones astronómicas y el detalle de la navegación. A menudo aburrida pues se limita diariamente a dar la estimación de la posición, pero de indudable valor al saber que la navegación media diaria era de 4 o 5 nudos, unos 225 kilómetros diarios, con grandes períodos sin vientos.

\footnotetext{
${ }^{1}$ Antonio PIGAFETTA, Primo Viaggio Intorno al Globo Terracqueo, prólogo de Emilio Lamo de Espinosa. REAL INSTITUTO ELCANO. Madrid. 2016

${ }^{2}$ Francisco DE ALBO, Diario del viaje de Magallanes desde la altura de San Agustín hasta regreso a España. Archivo General de Indias, Patronato 34, Rama 5.
} 
- Carta, De Moluccis Insulis de Maximiliano Transilvano al Arzobispo de Salzburgo.1522, se publicó en 1523 en Flandes.

- Declaración en Sanlúcar de los supervivientes, tras su llegada.

- Ginés de Mafra, existe copia del XVI de su libro: Libro que trata del descubrimiento y principio del estrecho que se llama de Magallanes.

- Antonio Britto, gobernador portugués de Ternate, carta al Rey don Manuel, una vez que hizo prisioneros a los que quedaron en Ternate, como luego narraremos.

- Instrucción Judicial de Sancho Díaz de Leguizamo. que se produjo al regreso ${ }^{3}$.

- Martín Fernández de Navarrete 1825-1837 recopilación de los documentos varios originales. Viajes y descubrimientos españoles en el Pacifico.

- Guillen Tato ${ }^{4}$, varios libros, mapas y artículos. Aunque su mapa tantas veces repetido muestra una línea directa de navegación, en el regreso desde las Canarias, cuando realmente hay que subir hasta las Azores y de allí al Cabo San Vicente.

- Libros de Amancio Landín Castro, sobre los Descubrimientos españoles del Mar del Sur.

\section{UNAS CONSIDERACIONES PREVIAS}

Que no van a gustar: la expedición es castellana y española (cántabros, astures, gallegos, vascos, castellanos, andaluces, junto a naturales de otras naciones) no hispano portuguesa.

Magallanes renegó de su lealtad a su Corona, (al no recibir una compensación económica de incremento salarial por heridas sufridas en 1514 en Fez) ${ }^{5}$ portuguesa y ésta le repudió por ello, e intentó por todos los medios abortar la expedición desde el inicio mandando dos flotas contra ella, la primera, que está en duda por los historiadores, para cortarles el paso al inicio y la segunda en las aguas del Pacífico.

La Corona dio órdenes expresas para evitar la entrada de portugueses en la expedición por razones obvias, aun así y en su contra, embarcaron 26 nacidos

\footnotetext{
${ }^{3}$ Archivo General de Indias.

${ }^{4}$ Julio F. GUILLÉN Y TATO. Cartografía Marítima Española, Editorial Naval 1943.

${ }^{5}$ Enrique MARTÍNEZ RUIZ, Desvelando Horizontes. Página 342. Fundación Museo Naval. 2016
} 
en Portugal- al servicio de Castilla - aunque la flota aragonesa y la anterior estaban unidas desde mediados del siglo XV. Portugal si aportó indirectamente la ciencia náutica, con la Escuela de Navegantes de Sagres, eso sí con inspiración de la escuela mallorquina y diversas aportaciones italianas y árabes.

La primera cuestión que planteaba la época, era el problema de la longitud: la esfericidad de la tierra era bien conocida desde hacía siglos, pero no el tamaño de su circunferencia -unos $90^{\circ}$ menos que los estimados-. De hecho, en la expedición, se llevaba un globo terráqueo de Benhaim aunque otros autores solo hablan de planos ${ }^{6}$. Ese fue el fallo de Colón que, pensó siempre que había llegado a las costas de la India, al mantener ese error del tamaño del globo. Aunque la descripción de los indios y habitantes distaba bastante de lo narrado por Marco Polo y los posteriores descubridores portugueses. Lo que debería haberle llamado la atención.

De la cuestión de la esfericidad de la Tierra encontramos lo primeros antecedentes en: Tales de Mileto que planteo seiscientos años a.C. la esfericidad de la Tierra, Arquitas de Tarento ( s IV a.C.) que la cifraba en unos actuales 74.000 kms. Arquímedes (s III): los estimaba en 55.500. Eratóstenes S.II a.C.) y el primer globo terráqueo de Crates de Malos (s II a.C.) con unos $46.250 \mathrm{kms}$. (250.000 estadios) ${ }^{7}$. Hiparco ( siglo II a. C.) resolvió la proyección sobre planos Posidonio de Apame: $44.500 \mathrm{kms}$. Y Ptolomeo se basó en ella. La actual es de 40.077 kms. en el Ecuador y 40.009 la longitud de un meridiano.

\section{LOS NAVEGANTES: SU ORIGEN}

Aunque el concepto de nación es posterior a la época, si se citaba, en los documentos de la expedición que, veremos a veces eran contradictorios en los números, el origen geográfico de los navegantes, así en la Real Cédula de 1519 se mencionan pormenorizadamente, por buque, el número de personas, nombre y oficio $^{8}$, con un total de 239. Pigafetta comentará que la tripulación, estaba compuesta por 237 hombres $^{9}$. Como las cifras son de la partida de Sevilla y no de Sanlúcar, ha de suponerse que en éste último puerto se embarcaron hasta el total de 271 que citan algunas fuentes. Y el origen de cada uno de ellos, de tal forma que la expedición estuvo integrada inicialmente por:

\footnotetext{
${ }^{6}$ Marcelino, GONZÁLEZ, La Nao Victoria, y su vuelta al Mundo. Fundación Alvargonzález, Gijón 2019.

${ }^{7}$ N. Del A. El estadio griego equivalía a 185 metros.

${ }^{8}$ Real Cédula de 5 de mayo de 1519, recogida en Desvelando Horizontes la Circunnavegación de Magallanes Elcano. Fundación Museo Naval. Madrid. 2016.

${ }^{9}$ Antonio, PIGAFETTA, Primo Viaggio Intorno al Globo Terracqueo. Obra citada, pág 16.
} 
- España, 171

- Italia, 35

- Portugal, 26 (pese a como hemos dicho las instrucciones en contrario de la Corona)

- Francia, 17

- $\quad$ Flandes, 4

- $\quad$ Rodas, 4

- Alemania, 3 (artilleros),

- Negros, 3

- Malaca, 1

- Inglaterra, 1 (artillero)

Por orden expresa de Magallanes no embarco ninguna mujer.

De ellos llegaron en 1522, 18 a Sanlúcar, quedaron 53-54, con la nave Trinidad (al mando de Gómez de Espinosa, debían volver vía Panamá y a punto estuvieron si no lo hicieron de descubrir la futura Corriente de Urdaneta, hoy Kuro Sivo) que permitía el retorno desde las Filipinas a las costas de California y Méjico). Se refugiaron en Ternate ya que la Trinidad estaba inservible para la navegación, allí fundaron una factoría y regresaron, tras su apresamiento por los portugueses, posteriormente a la llegada de Elcano a España, en 1527.

Durante el viaje se fueron incorporando nativos de diversa tierras e islas como Patagonia o Islas de San Lorenzo ${ }^{10}$, además de circunstancialmente numerosos nativos a modo de pilotos para ayudar en la navegación entre las islas que se iban encotrando, pero la mayoría morirían por enfermedad o muerte violenta.

Los oficios eran variopintos, destacando los capitanes, pilotos, maestres, marineros, artilleros, infantes, sobresalientes, aventureros, carpinteros, calafates, herreros, ...

\section{LA ALIMENTACIÓN}

Gracias al detalle de las declaraciones de lo que se embarcaba en cada nave, conocemos con extrema exactitud lo que se utilizaba como vituallas, para la

\footnotetext{
${ }^{10}$ N. A. San Lorenzo en honor del mismo por haberse recuperado las tripulaciones con los aprovisionamientos encontrados en esas islas desde el paso por el Pacifico, fue el nombre inicial que se dio a las luego conocidas como Filipinas.
} 
alimentación inicial, pues posteriormente se fueron nutriendo de la recogida de peces, aves, frutos y alimentos de las diversas tierras donde recalaron en su largo peregrinaje, donde el hambre y el escorbuto fueron constantes.

Precisamente el objeto de la expedición, la obtención de las especias, servían para, en la Europa renacentista, sazonar y conservar los alimentos. El precio de estas especias como la nuez moscada, canela, o clavo superaba en cinco veces el precio del mismo peso al del oro.

Las medidas de almacenamiento, reflejadas en los inventarios, eran las utilizadas en la época. Se hacía en en pipas (450 litros de agua), fanegas (55,5 litros), libras, arroba (1/4 de quintal unos 11,502 kg), ristra, celemines (4,6 dm3 en litros).

Lo embarcado inicialmente fue: vino, aceite, vinagre, bizcocho, pescado seco, tocinos añejos, habas, garbanzos, lentejas, harina, ajos (superada la ingenua creencia medieval de que quesos y cebollas alteraban las brújulas), arroz, quesos, miel, almendras en cascara, anchoas, sardinas, pasas de sal y lejía, cereales, pasas, higos, azúcar, carne de membrillo, alcaparras y mostaza.

En cuanto a los animales vivos que también se embarcaban: gallinas, vacas, cerdos, pájaros de diverso origen y a lo largo de la expedición, lo que se encontraba en cada lugar.

La alimentación abordo está perfectamente reglada en sus horarios, al igual que las instrucciones de uso de los fogones (preocupación del propio emperador en sus instrucciones) dado lo peligroso de las brasas. El fogón se colocaba en el castillo de proa, se encendía mediodía y se apagaba por la noche. Se hacían tres comidas diarias, en el desayuno bizcochos, vino ( el agua potable era un bien escaso y se deterioraba rápidamente), sardinas y tocino. En el almuerzo a las doce horas solares se consumían pescados, queso (espacialmente en las tormentas) o menestra de arroz con habas y garbanzos. En el ocaso del sol se repartía la cena consistente en más o menos la mitad de lo servido a mediodía. La carne se consumía dos veces por semana y los oficiales comían con manteles y cubiertos; mejor vino y bonito, en vez atún así como bizcocho blanco.

La regulación de la vida abordo era muy estricta y se remontaba, entre otros ordenamientos, al del Consulado del Mar y de las Siete Partidas de Alfonso X que, en numerosos epígrafes establecía decenas de reglas y normas que perdurarían a lo largo del tiempo. Y por supuesto las instrucciones y cedulas de la expedición. Se detallaba en ellas cómo eran por ejemplo las guardias, la primera al mando del capitán hasta las doce horas, la segunda desde las doce por el piloto que era relevado por el maestre hasta el final del anochecer ${ }^{11}$.

\footnotetext{
${ }^{11}$ Antonio, PIGAFETA Obra citada, página 16.
} 
El hambre sería demoledora, especialmente en la travesía desde el descubierto Estrecho de Magallanes hasta el avistamiento de las primeras islas del Pacífico, cuatro meses después y veinte muertos durante la singladura. Ratas a precio de oro y el cuero que forraba los mástiles fueron devorados por las tripulaciones hambrientas.

El escorbuto causo la mayoría de las muertes, aunque hoy sabemos que en las expediciones anteriores portuguesas, por el Índico, era conocido ${ }^{12}$ que la naranja y limones aliviaban la enfermedad, el problema era la obtención y conservación en buen estado de las mismas.

Respecto al material y equipamiento con el que fueron dotadas las naves, la lista es interminable : linternas, candados, azadas, ballestillas, fraguas, cera, cartas, compases, astrolabios, relojes de arena, globo terráqueo y otros. Respecto a la corredera las fuentes son contradictorias, unos dicen que es posterior a la epopeya y otros que ya se conocía.

En cuanto a la artillería era la habitual de la época (recordemos que la primera noticia de su uso fue en Barcelona en 1359 y el empleo de Bocanegra contra Pembroke $^{13}$ en 1372). Dedicaremos un apartado a ella.

Las dotaciones y mandos iniciales de los barcos fueron las siguientes:

- Trinidad: al mando de Magallanes, 62 hombres. Quedo en Ternate por inservible para la navegación.

- San Antonio: que desertaría al mando de Juan de Cartagena ${ }^{14}$ hasta su abandono en San Julián: 56 hombre que volvieron a España.

- Concepción: mando de Gaspar de Quesada con 44 hombres y Elcano. "Se tuvo que abandonada y quemada por falta de tripulación en Filipinas". 15

- Victoria: Luis de Mendoza y 45. "Abordada por los portugueses, que los perseguían desde las Molucas, y regreso a América".

- Santiago: Juan Serrano, 32 hombres. Que naufragaría.

- Total de la relación de gente de 1519 , resto sobresalientes y otros.

\footnotetext{
${ }^{12}$ Roger CROWLEY. El Mar sin fin. Ático de los Libros. Madrid 2018.

${ }^{13}$ N. A. Ambrosio Bocanegra en la Batalla de La Rochelle, derrotó al Conde de Pembroke.

${ }^{14}$ N. A. Juan de Cartagena fue nombrado Veedor General con el mismo nivel que Magallanes lo que ocasionaría más tarde los incidentes que comentaremos.

${ }^{15}$ Emilio LAMO DE ESPINOSA, Prologo: obra citada de Antonio Pigafetta.
} 


\section{LA CARTOGRAFIA DE LA ÉPOCA}

Aunque el término Cartografía no se empleó hasta el siglo XIX, nos referiremos a las artes de elaborar atlas, mapas, derroteros como tal en el artículo.

De toda la producción de la época apenas han quedado 30 cartas del siglo XIV y 150 del $\mathrm{XV}^{16}$.

Cabe destacar el absoluto desconocimiento de gran parte del Globo en la época, y parte se intuía en base a antiguos textos, suposiciones y leyendas ( Paraíso que se creía estaba en la tierra y por algunos se identificó en las costas de Brasil, la leyenda del reino cristiano de Preste Juan, Atlántida, San Brandan, Gog y Magog etc.). Pero en base a lo escaso de la producción cartográfica, esos hombres se aventuraron a la expedición por dos convencimientos y certezas; el primero que tras el viaje de Marco Polo se sabía que al otro lado de China, Cipango (Japón) había un mar que se suponía enlazaba con Europa. Ésta creencia fue en la que se empeñó Colón que, hasta su muerte pensó que había tocado las costas China. Pero ya inmediatamente después se tuvo conciencia de que o esas islas o un continente, debería tener un paso por el sur y otro por el Norte y que las tierras e islas descubiertas por Colón eran un continente intermedio entre Asia y Europa. Además las innumerables expediciones portuguesas a la India ya popularizaron que era posible y necesario viajar desde el Oeste hasta esas ansiadas islas de las especias.

Los primeros antecedentes cartográficos, por no ser exhaustivos en Estrabón hacia 64/63 a. C: que elaboró una geografía, basados a su vez en Eratóstenes e Hiparco con 17 tomos cuya primea impresión se conocería en 1479. Plinio el Viejo realizó una Historia de la Naturaleza con 37 libros y del segundo al cuarto dedicados a la geografía. Vegetius (IV d. C.) citaba de la necesidad de elaborar mapas para las campañas militares. Ptolomeo hacía 140 d.C., concluyó el Almagesto Astronómico y de Geografía con 27 mapas siendo traducido al árabe en el siglo IX en 1406 al latín y partir de 1477 se editaron ediciones de 4, 7 y 20 mapas de su Cosmografía.

A su vez Maquiavelo, Castiglione y Juan Luis Vives insistirían en la necesidad de elaborar mapas. Ibn Daid al Sahli y su hijo Mohamed detallarían un cielo con 1015 estrellas, Al Idrisi en Córdoba en el siglo XII dibujaría Sicilia y adivinaría la existencia de Guinea ${ }^{17}$.

En 1275 aparece la Carta Pisana desde el Mar Negro hasta Inglaterra, en 1296 el portulano más completo y antiguo del Mediterráneo.

\footnotetext{
${ }^{16}$ Dolores HIGUERAS, Marinos Cartógrafos españoles. Prosegur. Madrid 2002.

${ }^{17}$ Stephan ZWEIG, Américo Vespuccio relato de un error histórico. Acantilado, Madrid 2019.
} 
Aportación singular la de Alberti que vivió entre 1402 y 1472, con una topografía y cartografía en la que las medidas se realizaron mediante ángulos y triangulación ( recordemos que la de España, la diseñaría Jorge Juan en el siglo XVIII).

El italiano Toscannelli al servicio de Portugal elaboró (conocido por Colón) un mapa para la ruta de las Indias en 1474, aportando que la circunferencia de la Tierra era de $29.000 \mathrm{kms}$, similar a la indicada por Ptolomeo y dado que la uso Colón, nueva demostración del error de las tierras a las que había llegado. La escuela mallorquina tendría en Jefuda Cresques uno de sus grandes cartógrafos, se trasladó a Portugal, donde Enrique el Navegante ( por cierto nunca embarco en nada flotante) en el Cabo San Vicente instaló su Escuela de Navegantes. Los portugueses con el apoyo de los mallorquines elaboraron tres portulanos (de los que han llegado hasta nuestros días) anteriores a 1500, fecha de la creación del Armacen de Guiné ( precursora de nuestra Casa de Contratación, establecida en Sevilla en 1503) para en 1502 elaborar un Mapamundi. En 1519 aparece el Atlas Miller, con una conjunción de iconografía y cartografía. El judío salmantino, emigrado a Portugal, Abraham Zacuto ya había elaborado tablas estelares para ayuda a la navegación

Los portugueses fueron desde 1483, descubriendo territorios que aumentaban el alcance del mundo conocido; así en 1419 Madeira, en 1435 las Islas Afortunadas de los antiguos, en 1445 Cabo Verde y en 1486 la gesta de Bartolomé Díaz al dar la vuelta al Cabo de Buena Esperanza, para en 1499 Vasco de Gama consiguir regresar desde la India, en 1500 Cabral por error de navegación descubre Brasil, todo ello se plasmaría en cartas altamente secretas, cuya revelación llevaba la pena de muerte. Lisboa se convirtió en una ciudad de primer orden donde vivían decenas de cartógrafos astrónomos, dibujantes, aventureros...

Será, Américo Vespuccio, con sus de cuatro a seis hojas del Mundus Novus de 1503 (un éxito de impresión sin precedentes) el que dará lugar durante años al error histórico de atribuirle el descubrimiento del continente que por ello se bautizó con su nombre feminizado ${ }^{18}$. Costumbre que se repetiría al bautizar las Bermudas por su descubridor Juan Bermúdez, Fernando Poo por el mismo y Tasmania por Tasmán. Sería, Vespuccio, el primero en señalar que Colón no había llegado a la India y que se había topado con un nuevo continente interpuesto con Europa.

En 1506 en un anónimo ( atribuido a Bartolomé Colón) se publica una Carta Universal con las tierras descubiertas por Colón guardadas en tres croquis. ${ }^{19}$

\footnotetext{
${ }^{18}$ Stefan ZWEIG, Américo Vespuccio, relato de un error histórico. Acantilado Barcelona 2019.

${ }^{19}$ Ricardo CEREZO, La cartografía náutica española en los siglos XV, XVI y XVII. Madrid 1994 CSIC.
} 
En 1507, Waldseemuller dibuja el Planisferio, señalando la masa continental de Asia por primera vez.

Colón llevaría en su viaje distintos mapas pero especialmente el de D'Ailly, el Imago Mundi y el mencionado de Toscanelli.

Desgraciadamente en el terremoto de Lisboa de 1755 se perdió casi toda la producción cartográfica portuguesa.

El globo terráqueo de Martin Behaim supuso un hito histórico, al plasmar en el mismo mares y tierras, copia del mismo iría con Magallanes en su viaje. En él se recogen 48 banderas de naciones, 15 escudos de linajes y 48 retratos de reyes, todo cabía para rellenar los espacios y territorios y mares inexplorados sobre el cartón usado par elaborar el mismo.

La fabricación de los globos en la que años después, Mercator será el referente, era tarea complicada al no saber cómo proyectar las escalas, tema que sería éste quien finalmente lo consiguió y que todavía hoy es usada.

La escuela mallorquina, junto a la de varias ciudades italianas, como Génova, Amalfi, o Venecia, en el siglo XIII se ocuparon de la elaboración de los portulanos, que no eran cartas de navegación, si no la descripción de costas, puertos, islas y ríos con sus correspondientes códigos de colores. Será Ramón Llul en 1286 en su Maravillas del Orbe, uno de su grandes estudiosos al hablar de las propiedades de la aguja imantada, instrumentos de medida y descripción de cartas marinas. Y antes, Carlomagno se convirtió en un coleccionista de mapas dándoles una gran importancia estratégica. Los franceses tenían un portulano de 1270, que se elaboró para su rey Luis XI e ilustrarle en su viaje a Túnez. Angelino Dulcet en 1350 elaboró un portulano del Mediterráneo, conviviendo durante esa época la fructífera elaboración de mapas locales y regionales que se hacían en distintas abadías por Europa. En 1375 se publica el Atlas Catalán.

Hay que citar respecto a las mediciones que, aunque los romanos tuvieron un sistema cuadrangular local, de los terrenos la centuriazión ${ }^{20}$; las escalas y medias se perderían en la etapa medieval, donde los mapas eran a vista de pájaro y de aproximación. El mundo se representaba en un círculo con una "T" y los tres continentes alrededor de la misma.

Los ingleses disponían de su mapa de Hereford de 1450, un referente de la época; en 1472 se recogen las Etimologías de San Isidoro donde ya se citan los mapas.

Los alemanes por razones fiscales fueron reacios en esta época a la elaboración de mapas, temían los impuestos por posesiones que sus reyes o príncipes pudieran imponerles.

\footnotetext{
${ }^{20}$ José CASTAÑO, El libro de las pesas y medidas. La Esfera de los Libros. Madrid, 2015.
} 
En 1519 aparece una de las grandes obras hispanas, traducida a diversos idiomas, la Summa Geographica de Martin Fernández Enciso.

La producción de mapas tras el viaje se multiplicó por toda Europa aunque se basaba siempre en los relatos y mapas obtenidos de españoles y portugueses.

Los antecedentes de la cartografía cristiana son netamente musulmanes, serán ellos de los primeros en la traducción de textos griegos y romanos y la elaboración de centenares de obras de astronomía, historia, cartografía, sin las cuales el conocimiento del siglo XIV no hubiera avanzado. Destacamos a:

- Ibn Jaldún, tunecino de origen sevillano, el padre de la historia, según Toynbee.

- Abu-1- Qasim Maslama, Córdoba, revisión de las tablas astronómicas anteriores.

- Al Maqrizi (1364-1442) comentó la expedición genovesa a las Canarias en $1339^{21}$.

- Abraham Zacuto. Salamanca, al servicio de Portugal elaboró un Almanaque perpetuo.

- Ibn Abí Firás: tratado de derecho mercantil, con apuntes sobre el comercio marítimo.

- Ibn Al Saray: astrolabio Universal. Observatorio de Sevilla.

- Carta magrebiana, Granada 1330.

- Angelino Dulcert, detallaba los 32 vientos.

- Al Biruní: realiza la cartografía mediante observación astronómica.

- Al Idrisi ceutí, s XII. Libro de Roger.

- Al Himyani, esfericidad de la tierra ( conocida por los árabes) 24.00027.000 millas.

- Piri Reis, Turquía. S. XVI . ${ }^{22}$

\footnotetext{
${ }^{21}$ Al MAQRIZI, en Ibn Jaldún, El Mediterráneo en el siglo XIV. pág 52. Fundación el Legado Andalusí. Granada 2006.

${ }^{22}$ Piri REIS, El Libro del Mar, WEB Sociedad Geográfica Española. Madrid, 20 de junio 2019.
} 


\section{LA REPRESENTACION CARTOGRAFICA.}

Podemos afirmar que hasta el siglo XVIII, pero especialmente en el período que nos ocupa los periplos, los mapas, portulanos, derroteros, atlas, planisferios, mapamundis eran un arte en el que los autores irían rellenando espacios desconocidos, con mucha iconografía, incluyendo dibujos, desde la representación de los Reyes Magos en la Carta de Juan de la Cosa, pasando por animales fantasiosos en tierra y mares ( basados en el Polyhistor de Solinus, que a su vez utilizó la obra Naturalis Historia de Plinio el Viejo ${ }^{23}$ ), salpicado de referencias bíblicas ( no había que chocar con la Iglesia) decenas de islas en el Atlántico antes de Colón, incluso extraños homínidos sin cabeza o con mezcla animal. etc.

Las alegorías tienen un componente ideológico y religioso, al indio se le representa con facciones groseras y a los blancos como portadores de la civilización.

La mayoría de los grandes autores se basaban en cartas publicadas anteriormente, a las que añadían los nuevos hitos que aparecían fruto de las expediciones, en un verdadero terremoto cultural ya que, durante mil años desde Ptolomeo no se había realizado una explosión tan gigantesca de interés por los territorios que se iban descubriendo.

Imaginemos a los europeos de la época con sus viejas creencias y leyendas tomadas como certezas volando por los aires, ante la cantidad de noticias que llegaban: entre ellas que si había tierra y era habitada por culturas avanzadas más allá de las torres de Hércules, que la gente no se cocía al atravesar el Ecuador, y existía flora y fauna en el hemisferio sur, asimismo oriente era una tierra de lujos y exuberancias. Los productos de India o África salpicaban los mercados y todo esto apenas en dos generaciones desde mediados del siglo XIV a la primera Circunnavegación. Nunca hubo tanto interés por conocer el mundo y sus secretos. Una Europa renacida del oscurantismo bajomedieval y una Iglesia que asistía atónita a esos nuevos descubrimientos, a menudo con suspicacia e intervención de la Inquisición.

Los periplos, de los que existen numerosas noticias, eran en la Grecia a.C. relatos de navegación costera incluyendo observaciones de los pilotos las distancias navegadas, costas, corrientes...posiciones de los astros en el cielo, vientos, efectos del mar y otros ${ }^{24}$.

\footnotetext{
${ }^{23}$ Julio Cayo SOLINO, Colección de hechos memorables Gredos. Madrid 2001

${ }^{24}$ Ricardo CEREZO, obra citada, capitulo III.
} 


\section{CARTOGRAFIA ESPAÑOLA}

La cartografía antes, o arte de hacer los mapas durante y después de la Primera Circunnavegación se estructura en:

- Periodo griego.

- Periodo romano,

- Desconocemos en el periodo visigótico sus aportaciones si es que las hubo.

- Periodo medieval musulmán, la fuente de todo.

- Italianos y mallorquines en el Mediterráneo en el siglo XIV y XV.

- Renacentista. Europeos y musulmanes.

- Ilustrado.

- Para cada etapa, estudiaremos los protagonistas y sus obras correspondientes. Especialmente españoles.

- La Circunnavegación encontró otras formas diferentes de "cartografiar" los pueblos del Pacifico.

- Apenas 30 cartas del siglo XIV y 150 del XV.

- En el periodo ilustrado nuevos instrumentos de medición. Se acaban las fantasías, cartas trigonométricas. Rigor científico.

- Representaciones: Alpes como una pata de ave, Tajo: bastón rodeando Toledo.

- Siete Ciudades de Cíbola y reino de Quivira en La Florida.

- 1500 , carta de Juan de la Cosa, con Magos de Oriente...

- Antes de Magallanes-Elcano: creación de la Casa de Contratación 1503

- 1506. anónimo Pesaro tierras descubiertas por Colón

- 1519: Suma geográfica de Martín Fez. Enciso(sin mapas).

- mapamundis medievales: interpretación eclesiástica y social. Tiempo + espacio. mapa de Hereford.

- 1286 Ramón Llul Maravillas del orbe, cita la guja de imantada, cartas marinas e instrumentos de medida.

- 1375 Atlas Catalán 
- 1450.mapas rurales británicos

- 1472. Etimologia de San Isidoro.

- los portulanos ( solo mediterráneos) aparecen sobre 1279 con uno conservado de Luis XI para conocer el viaje que haría hasta Túnez. Pero no eran para planear rutas simplemente información de puertos.

- 1448: Mapamundi de Andrea Walsperger.

- Perspectivas a vista de Pájaro.

- $\quad$ se sabe que Carlomagno disponía de mapas de Constantinopla y roma

- planos de abadías, pero sin coordenadas matemáticas.

Autores como Maquiavelo, Castiglione, Juan Luis Vives, Elyot... escriben sobre la necesidad de los mapas. Da Vinci, Durero, fueron excelentes pintores de mapas.

- Alberti 1404-1472 publica la Topografía y cartografía con medición de ángulos.

- El mallorquín Jafuda Cresques se traslada a Portugal, como muchos mallorquines que serán imprescindibles $\mathrm{n}$ el avance de la cartografía portuguesa.

- 1492, Martin Behaim realizo un globo terráqueo (conocido y reproducido en el viaje de Magallanes-Elcano

- 1500, Armazen de Guiné. Introducción de las escalas por los portugueses.

- 1502, Mapamundi anónimo portugués.

- 1506. tierras descubiertas por Colón, Pessaro.

- Padrón Real: Enciclopedia Cartográfica de Américo Vespucio. El Padrón es una carta universal.

- $\quad 1511$ Cartas de Prunes y Salvat

- 1519, Atlas Miller y Summa geographica de Martin Fez. de Enciso, sin mapas

- Maximiliano I (1493-1519), gran aficionado a los mapas.

- Consejo Veneciano de los Díez ordena elaboración de mapas. 
- Nominalistas frente a neoplatonistas...Ockham los universales no existen no se presupone la existencia de objetos que no se han visto. Los universales existen en una realidad paralela.

- Países germánicos, reacios por razones fiscales (evitar los impuestos de reyes, príncipes...) a la elaboración de mapas.

\section{CARTOGRAFIA POST MAGALLANES - ELCANO.}

- La Circunnavegación supuso una explosión de interés por el conocimiento de la Corona de las tierras que iba descubriendo. La elaboración de cartografía fue continua tanto en los reinos ibéricos como en el resto de Europa, ansiosa de conocer los avances, las nuevas tierras, culturas etc. Fue una explosión de interés que revolucionó todo el continente. Las posesiones de portugueses y españoles fueron la envidia de los reinos europeas y una preocupación inmensa para Venecia que veía como quedaba desplazada del comercio con Oriente, con la aparición de estas dos nuevas potencias marítimas.

- Si primero fue el Armacen de Guiné portugués, la Casa de Contratación de Sevilla sería el faro que iluminaría le conocimiento durante décadas y la protagonista de esta labor de investigación, plasmación de descubrimientos, implantación de las escuela de pilotos, aplicando nuevos métodos y técnicas de elaboración cartográfica, que incluyó desde el uso masivo de la imprenta, nuevos tipos de papel, que sustituían las pieles bovinas etc.

- En el aspecto humano supuso una revolución al lanzarse los más atrevidos a la aventura americana y asiática.

La producción cartográfica tuvo sus autores y obras entre las que caben señalar las siguientes:

- 1522 , Nuño García de Toreno Filipinas y su Carta Universal.

- 1523, Carta Universal de Turín, origen Sevilla. Anónima

- 1525, Diego Ribero: Planisferio

- 1535, Francisco Falero, Tratado del Esphera y el Arte de Marear.

- 1526, Carta Universal de Juan Vespucio 
- 1527-29, Diego Ribero, otras tres cartas universales, apareciendo los descubrimientos de Pizarro en Perú.

- 1536-1544: Junta de Pilotos, grandes reformas de la cartografía.

- 1535, Alonso de Chaves, Carta de América, dibuja Yucatán como Península

- $\quad$ 1537, Pedro Nunes línea loxodrómica frente a la ortodrómica.

- $\quad$ 1542, Alonso de Santa Cruz, Mapamundi, más islario del planeta.

- 1542, Mapa Indias Orientales Jean Rotz. Francia.

- 1545 pedro de medina, Arte de Navegar del que dijo Guillen Tato: "ha publicado un verdadero tratado, el primero exclusivamente dedicado con profundidad ala Náutica...,25

- $\quad$ 1548, Jerónimo Chaves Chronografia o repertorio de los tiempos.

- 1550, Pedro de Medina: Suma de Cosmografía y Carta Atlántica de Diego Gutierres.

- 1551, Mapamundi de Martín Cortes, que indicaba la forma de realizar una carta de marear.

- 1551, Carta Universal de Sancho Gutiérrez.

- 1552, Regimiento de Navegación Pedro de Medina ( sistema de trazar origen de los vientos.

- Siguen apareciendo fantasías como San Brandán, Isla de la Eterna Juventud, grifos, cabezas de perro, hombres sin cabeza, animales monstruosos, Preste Juan, los Reyes Magos...

- 1556, Gerónimo de Girava: Dos libros de Cosmografía.

- 1570, Ortelius: Theatrum Orbis Terrarum. Amberes

- $\quad$ 1575, Escalante de Mendoza: Mapa de España que señalaba que era necesario: "entender aguja de marear y sus variaciones; saber usar la carta de marear Ballestilla y astrolabio; conocer los vientos y mareas, saber manejar el velamen según soplaba el ciento, tener noticias ciertas de tierras y puertos a alcanzar" ( se acabó la fantasía).

- 1580 , mapas de España, Francia e Inglaterra de Juan Rizo.

\footnotetext{
${ }^{25}$ Julio F. GUILLÉN, Europa aprendió a navegar en libros españoles. Instituto Histórico de Marina. Barcelona 1943
} 
- Problemas de la longitud, declinación magnética, inexactitud de los instrumentos, cuestiones de medición, especialmente por no controlar la velocidad.

En otros aspectos convivirán un espíritu "científico" con las leyendas, países imaginarios, bestiarios etc. espíritu de innovación de la Casa de Contratación, organización administrativa de primer orden. Cosmógrafo Real. Establecimiento de métodos de marcación, señalización, exámenes de pilotos...lo civil vs. papel de las ordenes especialmente jesuitas y agustinos.

- Exageraciones de los narradores. Fruto del desconocimiento de primera mano basarse en relatos más o menos verosímiles.

- La marcación del Tratado de Tordesillas antes y después del viaje y la posición de Cabo Verde, serán motivo de disputas que no se resolverían hasta mediados del siglo XVIII.

- Certeza de la esfericidad de la Tierra, el problema como hemos citado era la longitud de la circunferencia.

- Lo europeos se basaban en las publicaciones españolas y portuguesas.

- El mérito hispánico fue el salto a lo desconocido, la navegación en alta mar, no costeando. Continuo interés por la búsqueda de pasos, tierras incógnitas con recursos humanos limitados.

- Elaboración de los Regimientos de Navegación, almanaques y efemérides, que establecen la posición de los planetas.

\section{LOS VIENTOS}

Evidentemente no se puede navegar oceánicamente, a vela, sin servirse de los mismos. Ya los griegos utilizaron una rosa de los vientos desde el norte en la parte superior y en sentido de las agujas del reloj nombrados estos como: Boreas, kackias, apeliotes, euros, notos, lips, céfero y skim para en el siglo XIII los ocho vientos del Mediterráneo: tramontana, greco, levante, sirocco, osho, lebeccio, poniente y maestro, hasta finalmente los 32 .

Será el descubrimiento de los vientos ( alisios) que bajan de las costas de la península hasta África para, después remontar hasta el Caribe, uno de los principales acontecimientos, las terribles calmas del mar, tanto en el Atlántico como en el Pacifico, serían la desesperación de los navegantes, pues perdidos los 
vientos, el avance o el regreso eran imposibles. Así que la combinación de uso de los vientos, observación de las mareas, y de movimiento de los planetas serán los conocimientos básicos para emprender la aventura oceánica. Es lo que se recogerá en los llamados regimientos de navegación.

\section{LAS APORTACIONES “TECNOLÓGICAS”.}

Además de la cartografía otras técnicas y tecnologías destacaban en la época de la circunnavegación. Así la construcción naval donde los desarrollos autóctonos convivían con la copia o adaptación de métodos utilizados en otros mares será una constante y abarcará desde el empleo de naves como las urcas de origen nórdico, el uso de la nao y la carabela que permitían el almacenaje de provisiones y aventurarse en mares adversos. Habrá decenas de tipos de embarcación tanto de origen árabe, como latino y de origen nórdico.

No existían reglas de construcción ni modelos en serie, ni nada que se le pareciese, ya que el oficio de los carpinteros de ribera se trasmitía generacionalmente de forma verbal. Habrá que esperar a Gaztañeta, en España, a finales del XVII para tener manuales de construcción en serie. A medida que se apreciaba lo que ocurría con los distintos tipos de embarcaciones en la navegaciones oceánica la experiencia náutica pronto terminó con los altos castillos de proa y popa mucho más tarde, con las incomodas cofas en el bauprés y otros elementos engorrosos, al comprobar la resistencia al viento, durante la navegación.

La aparición de la broma, sería una desgracia pues perforaban los cascos haciéndolos rápidamente inservibles, se tardarían dos siglos en forrar la obra viva con cobre. Aunque ya en la expedición la obra viva (parte del casco sumergida) iba protegida. Igualmente se sustituyeron las popas redondas por planas. Y la armazón externa en tingladillo, se reemplazó por la unión de los tablones con estopa y alquitrán o brea como junta aislante.

Al no existir modelos para su reproducción en serie, cada carpintero de ribera y zona fabricaba sus propios barcos. Rafael Monleón ${ }^{26}$ pintor naval del Museo de Madrid en el siglo XIX elaboró tres volúmenes en los que pintó un millar de embarcaciones de todo tipo históricas. Desconocemos en muchos casos las fuentes en las que se basó. Pero debemos citar las carracas, carabelas, naos, zabras, urcas, cocas, galera, bagula, bucio (galera comercial), carabo, carauzel, fusta, coqueta, patache, haloque, saetia, sambruk, Taridas, Uxer (para caballos)...los daus del Indico los juncos y bignadays se descubren en las Molucas.

\footnotetext{
${ }^{26}$ Rafael MONLEON, Construcción naval. Lunwerg. Barcelona 1990.
} 
La aportación de maderas africanas y americanas, rápidamente se impusieron por ser mas consistentes y de larga duración conviviendo con las peninsulares en partes de los buques.

Otra gran innovación del periodo previo a la aventura, fue la adopción de la vela latina ( que no tiene nada de la misma pues es importada por los árabes desde el Indico), que permite tomar viento de varias direcciones y no solo desde la popa, como hacen las velas cuadras. La sustitución del timón manual por el unido al casco, son aportaciones europeas. Las del Pacífico eran entre otras el empleo de hojas de palma y piña para la confección de velas, los cuadrantes de mareas tan populares en la Polinesia, la observación del cambio del color del mar ante las costas o sencillamente el vuelo de los pájaros.

Sin duda entre las técnicas de navegación y orientación será la brújula el compás las más preciada al poder orientarse con el polo magnético. Del origen del compás o brújula se ha escrito mucho, desde su posible origen mogol, pues las caravanas de camellos ya lo usaban, pasó a China ${ }^{27}$ y de allí al mundo árabe. Los italianos de Amalfi se consideran sus descubridores y los ingleses del siglo $\mathrm{XV}$ igualmente. Seguramente, como ocurre en otros campos tecnológicos, puede que simultáneamente se fueran usando y descubriendo el uso de las agujas imantadas de la magnetita.

En la expedición serán las agujas que serán un gran problema al navegar Magallanes por los mares del sur, ya que está perdía su efectividad. Se encontraron con el cambio de sentido de corrientes y vientos y nuevos cielos con estrellas desconocidas como la Cruz del sur.

Otro instrumento imprescindible de la época para orientarse en tierra o mar era el astrolabio que tiene un origen muy remoto, en el siglo III a. C. ya era citado por Ptolomeo y fue perfeccionándose por los árabes en la península ibérica y en el siglo XV se extendió por toda Europa. Complejo de utilizar, se iría sustituyendo por otros instrumentos de más fácil aprendizaje y uso.

La ballestilla bien conocida en Europa era otro instrumento pero su empleo abordo era impreciso con errores de 4 o 5 grados, que proyectados a larga distancia ocasionaban errores de bulto ${ }^{28}$ en la estimación para situarse.

Por su parte las esferas armilares, eran complejos globos con aros varios que señalaban el movimiento de planetas, astros e función de la posición geográfica elegida y la época del año.

\footnotetext{
${ }^{27}$ Alan GURNEY. El Compás. Editorial Juventud. Barcelona 2005.

${ }^{28}$ CROWLEY obra citada.
} 


\section{PRINCIPALES PERSONAJES.}

Además de Magallanes y Elcano, de los que no resumiremos aquí sus birografías, tenemos que citar en primer lugar a Diego Barbosa portugués afincado en Sevilla, Ruy Falero, portugués también en Sevilla, cosmógrafo, astrónomo, nigromante, será el que daría el impulso inicial a la idea del viaje, sin él y su gran influencia en la Casa de Contratación no hubiera sido posible. Por supuesto el rey portugués Don Manuel que protagonizo los esfuerzos portugueses de múltiples expediciones, y que sin embargo intento abortar la aventura de Magallanes, al que ya había humillado negándole un incremento de sus rentas en unos ducados tras ser herido en campaña en el norte de África y que sería el desencadenante del entonces ya intrépido navegante (había llegado a las Indias) que Magallanes se refugiara en la Corona española y por sus intentos de abortar la expedición, antes y durante la misma, aunque habría que revisar las fuentes pues no está muy clara la primera expedición para abortar el viaje.

El emperador Carlos I, informado de la iniciativa la apoyó decididamente desde el primer momento, financiando en gran parte la misma y cuidando hasta el último detalle la gestación de la misma. Impartiendo órdenes, directrices, cédulas que detallaban en todo lo que se pretendía, entre otras cosas, no chocar con el rey de Portugal, realizar acciones de hermanamiento y no sometimiento con los pueblos que se fueran encontrando por la ruta, respetando en todo sus bienes, mujeres etc. y circunscribiendo la operación a una aventura comercial como ya hemos citado.

El burgalés Cristóbal de Haro, fue el cofinanciador del viaje, en lo que hoy llamaríamos una financiación público- privada. Fue apoyado por otros comerciantes burgaleses y castellanos hasta el 39 por ciento ${ }^{29}$ del total de coste de la expedición. Hay que recordar que el Consulado del Mar de Burgos tenía casi monopolio de la exportación vía puerto de Santander del comercio con Flandes y desde allí al resto de Europa, y Castrojeriz ${ }^{30}$ era una de las principales villas de esa organización comercial, con factores y oficinas de representación en Amberes o Brujas, querían tener lógicamente capacidad de transportar las especies que vía terrestre desde Sevilla fueran llegando.

\footnotetext{
${ }^{29}$ Nota: dato aportado en la Conferencia del Rector de la Universidad de Burgos en el Rectorado el día 31 de mayo de 2019.

${ }^{30}$ Marta NEGRO y otros. De Castrojeriz a Brujas. Edición Parroquia de Castrojeriz. Burgos 2010
} 


\section{LA ORGANIZACIÓN NAVAL Y NAVIOS}

Mucho se ha debatido sobre el tipo de embarcaciones empleadas, pero si nos atenemos a los documentos de la época, se habla de cuatro zabras ${ }^{31}$ y una carabela $^{32}$ :

Estas eran la San Antonio, de 144 toneles $^{33}$; la Trinidad, 132 toneles; la Concepción de 108; la Victoria de 102 y la Santiago de 80 toneles.

Las instrucciones de la organización obligaban, entre otros detalles a por las tardes realizar salvas de cañón, los pilotos tomaban las alturas y puntos con la ballestilla y astrolabio y los escribanos asentaban lo que decían los pilotos. Estos eran los verdaderos expertos en la navegación, pues a menudo los capitanes eran los armadores o mandos pero sin conocimientos tan especializados. Pasaban un examen duro en la Casa de Contratación de Sevilla, que superado los homologaba como tales.

Se navegaba en convoy procurando no perderse de vista, utilizando el cañón y señales luminosas para no perderse. Aunque a menudo se enviaban uno o dos navíos más ligeros, como avanzada y reconocimiento.

La organización fue plasmándose en diverso hitos entre los que destacamos:

- 20-1-1518:comitiva a Valladolid para ver a Carlos V.

- 23-II-18: escritura pública ante escribano de la Corte.

- 22-III-18: Capitulaciones de Valladolid, capitanes: Falero (finalmente no embarcaría) y Magallanes. Cristóbal de Haro financia parte de la expedición: suministrando cobre, azogue, sedas...

- 24-X-18 carta de Magallanes señalando dificultades de armar la expedición.

- 8-V- 19: Instrucciones Carlos V: solo navegar en los límites castellanos. Registro General de la Expedición.

- 5-V-19: Real Cédula de personas y cantidades de cosas a llevar. Relación de Gente.

- 30-III-19: nombramiento Veedor General: Juan de Cartagena. Lo que ocasionaría graves problemas en el gobierno del viaje. De facto el mando era bicéfalo, actuando el Veedor al mismo nivel que Magallanes, dando lugar a los primeros incidentes graves del viaje al no comunicar

\footnotetext{
${ }^{31}$ Zafra: embarcación atlántica para uso comercial de unas 200 toneladas.

${ }^{32}$ Carabela de tres palos y unas 300 toneladas.

${ }^{33}$ Tonel: dos pipas de 27 arrobas y media.
} 
Magallanes al mismo y resto de capitanes algunas de su decisiones iniciales de cambio de ruta, lo que motivo la rebelión de los castellanos y la dura respuesta de Magallanes que incluyó algunas ejecuciones.

- $8-\mathrm{V}-19$, Instrucciones a Magallanes.

- 9-VIII-19: se realiza un cuestionario a las personas que debían contar las motivaciones para el viaje. Era un largo interrogatorio personal a cada miembro de las tripulaciones.

- 24-VIII-19: Donación del hábito de Santiago (mejor símbolo de su españolidad no cabe) y 15.000 maravedíes al Convento de $\mathrm{N}^{\mathrm{a}}$ Sra. de la Victoria, por parte de Magallanes, lo que demuestra el convencimiento íntimo de su misión, su altura humana y espiritual.

- 10-VIII-19: salida del puerto de las Mulas. Sevilla.

- 24-VIII-19: testamento de Magallanes.

- 20-IX-19: Sanlúcar. Salida tras las últimas operaciones de aprovisionamiento y logística no exenta de numerosos incidentes, como corresponde a una empresa de tal magnitud, reivindicaciones salariales de los tripulantes, nuevas incorporaciones de tripulaciones, estafas en los abastecimientos etc.

- 26-IX, llegada a Tenerife, navegación eludiendo costas de Guinea, posesión portuguesa, adentrándose en el Atlántico sin seguir las instrucciones recibidas por el emperador y que daría lugar al amotinamiento posterior como hemos dicho de de Juan de Cartagena y varios mandos.

- 3-X-19, Paso cerca de Cabo Verde

- 13-XII-19, entrada en la Bahía de Guanabara, y el hoy Río de Janeiro. Permanece 13 días.

- 31-III-20, Puerto de San Julián: rebelión de Juan de Cartagena, y otros, apuñalamiento del tesorero Luis de Mendoza, afín a Magallanes y ajusticiamiento de tres rebeldes. Naufraga la Santiago en mayo.

- Navegación por el hoy Rio de la Plata ansiado paso por el sur pero al descubrir q avistamiento de Montevideo, se continua la navegación al comprender que es un estuario de un río y no el ansiado paso. Se continua bajando hasta el estrecho que llamarían de las Once Mil Virgenes el 21 de octubre de 1520. Con varios incidentes, entre ellas la deserción y regreso a España de la San Antonio, al mando de Esteban Gómez, que según el relato 
de Pigafetta odiaba a Magallanes. Pero antes una estancia larga hasta finalmente desembocar, tras pasar el entonces llamado Estrecho de los Patagones, en el mar denominado como Mar Pacífico ( el 28 de noviembre de 1520) de allí subieron, hasta las primeras islas, en una navegación penosísima de tres meses y veinte días.

- Llegan a la Isla de los Ladrones (Guam) llamadas así por haberles robado los nativos un esquife y donde tuvieron que combatir contra ellos. Parten el 9 de marzo de 1521.

- Se descubren, entre otras muchas, Zuloan, Massana, Humunu, islas Marianas, Guanjan, San Lázaro del que no se tenía conocimiento, siendo la antesala del archipiélago del mismo nombre, posteriormente bautizadas como Filipinas. Se llega a Sri Lanka, Sualan, Samar, Cebú, Palawan, Joló, Cagayan, Limasawa, Quipit, Sibuco, Boigo, Virano Bataloque, Candicar, Sarangani Borneo, Timor...

- $\quad 7$-IV llegada a Zubu, y cerca Mactán.

- 28-IV, Magallanes fallece en combate, por su imprudencia en Mactán. Se tiene conocimiento de la cercanía de las Molucas.

- Se toma rumbo hacía Palaoán. Permanecen 35 días en Borneo y 43 en Cimbómbom. Después tomaran la ruta a Mindanao.

- 26-X, Biraham Batolach. Y de allí a las Molucas, objetivo del viaje al ser las productoras de las especias.

- 8-XI, entrada en Tidore, el 11 están en Ternate, en las islas se aprovisionan de clavo, nuez moscada, jengibre y se encuentran con un portugués, Alfonso de Lorosa, superviviente de expediciones portuguesas anteriores.

- 21-XII salida de Tidore, con apoyo de pilotos locales. Abandonando la Trinidad, quedándose Juan Carvallo con 53 hombres.

- 25- salida de la isla de Malluá, tras haber recorrido otro largo número de islas. De allí a Timor y Malaca.

- Varios cambios de capitanes hasta que Elcano tomó el mando, regresando durante cinco meses de navegación hacía España, con el incidente de Cabo Verde, donde los portugueses por un error de los navegantes hispanos, que botaron un esquife de la Victoria, pretendieron engañarles diciendo que provenían desde costas americanas, pero al pagar las 
provisiones suministradas con clavo algunas provisiones descubriendo los portugueses el engaño.

- Quedaron, como señalamos antes, por no poder usar la Trinidad, 53 hombres que fueron finalmente apresados por el portugués Antonio Britto en Ternate, donde habían fundado una factoría. Regresarían entre otros Espinosa Mafra y Pancaldo que informarían en 1527 en Valladolid de su particular odisea.

- Llegan a Sanlúcar, tras casi tres años, el 6 de septiembre de 1522, 13 españoles, tres italianos ( entre ellos Pigafetta), un portugués y un alemán. Habría que ver la cara que se les quedaría a los desertores de la San Antonio, al ver que la expedición finalmente había concluido con éxito.

\section{CIENCIA NÁUTICA}

El conocimiento para navegar se basaba en los "cuatro términos": RUMBO, DISTANCIA, LATITUD y LONGITUD, podemos añadir el de PROFUNDIDAD. De ellos el de la longitud sería el quebradero de cabeza sin resolver hasta la aparición a mediados del siglo XVIII del reloj de Harrison.

- AGUJA DE MAREAR, sirve para señalar el rumbo del norte magnético, no el verdadero.

- ASTROLABIO, para indicar la latitud, con dos tratados de origen musulmán: el de Ibn al Samah (980-1034) y el de Ibn al Saffar (H 1034). ${ }^{34}$

- ESFERA ARMILAR, ya descrita.

- 1514 Johannes Wener, establece el sistema de navegación por el movimiento de la luna, aunque ya era conocido.

- CORREDERA, para medir la distancia. Aunque su existencia es posterior parece que algún tipo de dispositivo similar ya se empleaba, además del sistema de tirar virutas por la proa o un tonel y al llegar a la popa medir el tiempo que había recorrido.

- AMPOLLETA o reloj de arena, instrumento imprescindible ya que media el tiempo navegado y señalaba los relevos de las guardias.

\footnotetext{
${ }^{34}$ Mercedes GARCÍA-ARENAL (coord..) Al Andalus Allende el Atlántico. Pág. 45.Unesco. Granada 1997
} 
- SONDA, de origen portugués, especifica la profundidad, el tipo de fondo, al tocar la plomada del extremo aquel, untado de sebo recogía, arena, plantas o polvo de roca del fondo marino.

- ANCLAS, de la piedra agujereada en el centro preromana a las anclas y rezones de hierro. El uso de ellas al fondear por debajo del Trópico cambiaron, no se conocía todavía el efecto de Coriolis, pero ya se entendía los cambios de diferente rotación que se producía según la posición en un hemisferio $u$ otro.

- MATERIALES y MADERAS, de la más variada índole y orígenes diferentes: pino, caoba, roble, cáñamo, lino etc.

- $\quad$ ATLAS, mapas, mapamundis...

- LONGITUD: hasta HARRISON en 1736 con su reloj no fue posible obtener la misma con exactitud, se hacía por estima.

- $\quad$ RUEDA DE ALTURAS DE LA POLAR. De Valentín Fernándes en 1518.

\section{LEGISLACIÓN}

Existía ya abundantes normas que regulaban tanto la vida en los barcos, como el comercio, costumbres, tráfico marítimo etc. las principales:

- TRATADO DE TORDESILLAS, con el error de no marcar debidamente qué isla de las de Cabo Verde era desde la que se medía el meridiano. Sería la mayor preocupación de los navegantes no incumplir el mismo por orden expresa del emperador.

- $\quad$ SIETE PARTIDAS, de Alfonso X El Sabio en múltiples capítulos detalla innumerables aspectos de los citados.

- CONSULADOS DE MAR, organizaciones de origen italiano tenían una misión jurídica y mercantil. De los españoles caben señalar los de Barcelona, Mallorca, Valencia, Barcelona y Burgos. A su vez el Libro del Consulado del Mar.

- Hermandad de las Cuatro Villas y la de las Marismas, organizaciones administrativas medievales, de carácter administrativo con profusión de instrucciones, fueros, etc.

- INSTRUCCIONES Y REALES CÉDULAS, elaboradas por Carlos V, recogían infinidad de temas a acatar por los capitanes de la expedición. 


\section{ALGUNOS USOS Y COSTUMBRES EN LA MAR}

Tipología: ya hemos descrito antes los diferentes oficios de los que se incorporaron al aventura.

- Salarios, fijados de antemano antes del embarque, suscitaron quejas de los expedicionarios al Emperador. Estaban por encima de lo que cobraban los marinos de la época, recordando que entonces no había diferencia entre marinos de guerra y mercantes.

- Castigos: aunque los castigos físicos eran considerados infamantes y se utilizaban con cautela y en caso extremo se podía ahorcar, colgar los cuerpos de las vergas, como medida ejemplarizante, desmembramiento, destierro especialmente para las personas notables, reducción de salario o de racionamiento en las comidas.

- Recompensas: estas principalmente era la participación en el reparto de los botines y en la venta de la carga confiscada. También medallones colgantes de oro y cadenas. Tan habituales en nuestros soldados de los tercios.

- Fogón: con los cocineros se situaba en el castillo de proa se encendía a mediodía y se apagaba por la noche.

- Oficiales: comían en mesa, con mantel y cubiertos, mejor vino y bonito en vez de atún y bizcocho blanco que la marinería.

- Carne se comía, cuando la había, dos veces por semana, dos litros de agua diaria, mezclada con vino a menudo y queso especialmente en días de tormentas.

\section{ARMAMENTO Y MUNICIÓN}

La artillería de la época, compuesta por piezas heterogéneas y de diversos calibres, que cada fabricante realizaba según sus criterios. Todas las armas eran de ánima lisa.

Las primera fuentes europeas citan el empleo de la artillería naval en 1359 en Barcelona y en 1372 en los combates de Bocanegra frente a Pembroke.

Las piezas eran de hierro o bronce de fundición. Podemos nombrar:

- PEDREROS. $75 \mathrm{~cm}$ y $3,7 \mathrm{~cm}$ de boca de salida y en horquilla. 
- ARCABUZ, que medía entre 29 y $171 \mathrm{cms}$, se utilizaba en las bordas y con horquilla en tierra, fue sin duda el arma más temida por los pueblos que no lo conocían.

- BOMBARDAS, precursoras del cañón, construida por dos piezas separadas, el cuerpo de la misma y los aros de hierro que lo reforzaban externamente.

- CAÑON, pieza que disparaba balas de 24 a 56 libras.

- FALCONETES, con mayor longitud que el pedrero y boca de 5-7 cm.

- SACABUCHE, del tipo culebrina, arma larga precursora de las armas portátiles.

- CULEBRINAS, pequeños cañones que cargaban una bala de 16 a 30 libras. $^{35}$

- SACRE, o cuarto de culebrina.

- BALlestas y ARCOS, Armas individuales cuyo uso certero era demoledor entre las filas de los enemigos.

- ARMAS DE FUEGO PORTATILES: arcabuz entre 129 y $171 \mathrm{cms}$. Sería el arma por antonomasia, temida por su ruido y la precisión a corta distancia de sus balas.

- COSELETES y ARMADURAS. A raíz de moverse en zonas tropicales y húmedas se introdujeron petos de tejido contra flechas, liberándose de las pesadas armaduras.

- MUNICIÓN: HIERRO y PIEDRA. Se fundían las primeras abordo.

- FUEGO GRIEGO, no se utilizó.

\section{CHINA, INDIA Y JAPÓN, ENTONCES}

Nuestra errónea visión histórica eurocéntrica ha olvidado que en el otro lado del planeta existían en la época culturas y civilizaciones tan o más avanzadas que la nuestra.

Las expediciones portuguesas desde Almeida y de Alburquerque habían ya iniciado su posicionamiento en lugares como Goa, Conchin, Ormuz, etc. por lo que los occidentales eran conocidos y no precisamente muy gratamente en India, La Meca, Egipto...

\footnotetext{
${ }^{35}$ Antonio DE SOUSA, Historia de la Artillería, De Re Militari, sin fecha.
} 
- En China, gobernaba la dinastía Ming (1359-1644): cuya principal hito fue construcción de la Muralla, y la organización del Estado. Habían abandonado las gigantescas expediciones comerciales, con el culmen con las de Zheng $\mathrm{He}^{36}$.

- En la India: Imperio Vijayanagra ( 1336-1565) y entre 1509 y 1529 el reino Krsna Deva Raya, documentado por el portugués Paes y otros: con expansión territorial, numerosas expediciones marítimas comerciales, impulso tecnológico y expansión del hinduismo. Pero la llegada portuguesa alteraría los equilibrios políticos y comerciales de las diversas ciudades y puertos.

- El shogunato Ashikaga (1338-1573), dominaba Japón. Época de guerras civiles entre los antiguos propietarios y los nuevos condestables imperiales, y establecimiento de un régimen militar; no contribuyó a ninguna expedición naval o militar fuera del territorio del Japón.

- En Cebú, Malaca, Borneo: numerosos reinos en guerra unos con otros. Muchos juraron fidelidad a Castilla y pronto formaron parte de la Corona: Filipinas, Carolinas, Marianas..

\section{CONCLUSIONES DEL VIAJE}

Lo aportado por el viaje tuvo consecuencias que cambiaron la historia de la humanidad, entre ellas:

Se constató que el océano ocupa la mayor parte del globo, al determinarse la mayor amplitud de la circunferencia de la Tierra. De los $27.000 \mathrm{kms}$ a algo más de 40.000 .

La ansiada búsqueda de un paso por el sur del continente americano se encontró en el estrecho hoy llamado de Magallanes (tuvo hasta 11 nombres distintos) y supuso encontrar el camino hacia las indias por el oeste. Otros desde Inglaterra intentarían también, sin éxito descubrir el paso del norte.

Descubrimiento de la Cruz del Sur como orientación de los navegantes en ese hemisferio.

El magnetismo terrestre se comprobó que variaba y por ello se introducirá un método de cálculo de los desvíos de la aguja.

Se certifica en el regreso de Elcano por al pasar por Cabo Verde que hay un día de variación en la circunnavegación.

\footnotetext{
${ }^{36}$ Michael YAMASHITA. Zheng He, los 7 viajes épicos alrededor del mundo del mayor explorador chino (1405 - 1433). Editorial Blume Barcelona 2007.
} 
Se descubren nuevos medios de orientarse en la mar, sorprendiendo que el método de las distancias lunares ya era conocido en el Pacífico, cuando Magallanes llegó por el oeste.

La aportación previa a la expedición de los cartógrafos mallorquines, hispanoárabes y portugueses constituyen una aventura científica de primer orden. $\mathrm{Su}$ resultado como hemos analizado cientos de mapas, cartas, y portulanos.

Se enriquece el vocabulario con nuevas palabras como canoa, hamaca ( que empezaría a utilizarse desde entonces en todos los navíos, denominándose coy), así como el inventario de cientos de especies vegetales, animales y diferentes culturas.

Tras diversas encuentros entre españoles y portugueses y la discusión sobre la línea del Tratado de Tordesillas, se acordó que las Molucas fueran para Portugal y las Filipinas, (Acuerdo de Elvás en 1526) para España.

Se establecen alianzas comerciales, no de sometimiento a la Corona, con gran número de reinos de las islas aliados que perdurarían con las Carolinas y Marianas hasta finales del siglo XIX.

España se hizo dueña del Pacífico durante dos centurias, océano al que se le denominaba "el Lago Español" y salvo incursiones puntuales de franceses, holandeses e ingleses nunca se perdió la ruta desde Filipinas a México, basada en el sistema de flotas del Galeón de Acapulco. De unos 450 navíos, que hicieron esa ruta se perdieron tan solo cuatro. El Galeón supuso el establecimiento de acuerdos comerciales con China y Japón en lo que puede considerarse la primera globalización del planeta.

La aportación española a la ciencia náutica en cosmografía, cartografía, astronomía y navegación es indudable, pudiendo decirse que el resto de marinas aprendieron a navegar en nuestros libros, como señaló acertadamente Guillén Tato, en su libro ya citado.

El desarrollo de nuevos instrumentos náuticos o mejor el perfeccionamiento de los que hemos narrado, constituyó una revolución sobre todo en Inglaterra y en Europa Central.

El principal problema era qué instrumentos y cómo construirlos cuando no se sabía medir y las medidas eran un verdadero galimatías en la España de la época (no se normalizarían hasta 1850 con la introducción del sistema métrico decimal).

Se impone el empirismo y carácter científico frente al pragmatismo anterior, aunque el método de prueba y error era lo predominante. Los métodos de observación de movimientos de planetas, la luna, estrellas, la aplicación de diferentes métodos de cálculo irían evolucionando lenta pero contantemente. 
Se produce una explosión de expediciones y viajes que abarcaron toda la periferia y gran parte del interior de América, así como islas tan remotas como Guam.

El prestigio para las Españas fue innegable en toda Europa, convirtiéndola en la potencia de la época casi un talasocracia, y más posteriormente con la unión de los reinos ibéricos con Felipe II.

\section{BIBLIOGRAFIA}

Alfredo ALVAR, Juan Sebastián Elcano. La Trebere Madrid, 2016

Rubén ALVAREZ, Cartografía y Navegación, Mastergraff, Montevideo,1996.

Salvador BERNABEU, Las Expediciones Maritimas Españolas. Ed. Lunwerg, Madrid, 2000.

David, BUISSERET, La Revolución Cartográfica en Europa. Ed. Paidós. Barcelona, 2003.

Ricardo CEREZO, La Cartografía Náutica española en los siglos XIV, XV y XVI. CSIC. Madrid.1994

Mercedes GARCÍA-ARENAL. Al Ándalus Allende el Atlántico. UNESCO. Granada 1997.

Julio F. GUILLEN, Europa aprendió a navegar en libros españoles. Instituto de Marina. Barcelona. 1943

Alan, GURNEY: El Compás, Ed. Juventud. Barcelona, 2005.

María Dolores HIGUERA: Marinos Cartógrafos españoles. Recopilación varios textos. Ed. Prosegur. Madrid. 2002.

Paul, LAXTON, La nueva naturaleza de los Mapas. Ed. FCE. México, 2005.

Antonio LÓPEZ y Carmen MANSO PORTO, Cartografia Española del Siglo XVIII, Tomás López en la Real Academia de la Historia. Real Academia de la Historia, Madrid 2005.

María Luisa, MARTÍN-MERÁS, Cartografia Maritima Hispana. Ed. Lunwerg. Madrid 1993.

Enrique MARTÍNEZ RUÍZ (coordinador.) Desvelando Horizontes, La circunnavegación de Magallanes y Elcano. Fundación Museo Naval. Madrid. 2016.

Vicente PALACIO ATARD, (coord.) "España y el Mar en el siglo XVIII". Edición Privada. Marinves, S.A. Sonchika, 1999.

Antonio PIGAFETTA, Primo Viaggio Intorno al Globo Terracqueo, Madrid. Real Instituto Elcano, 2016.

María Jesús Viguera Molins, Ibn Jaldún, el Mediterráneo en el siglo XIV. Fundación Legado Andalusí. Granada, 2006

Manuel, SELLES, Instrumentos de Navegación. Editorial Lunwerg, Madrid, 1994.

Dava SOBEL, Longitud. Editorial Debate 1997, Madrid. 